\title{
Medicinal plants use in central Togo (Africa) with an emphasis on the timing
}

\author{
Tchadjobo Tchacondo, Simplice D. Karou, Amégninou Agban, Mamouda Bako, Komlan Batawila, \\ Moctar L. Bawa', Mensavi Gbeassor, Comlan de Souza
}

Centre de Recherche et de Formation sur les Plantes Médicinales (CERFOPLAM), Université de Lomé and ${ }^{1}$ Laboratoire de Chimie de l'Eau, Faculté des Sciences (FDS), Université de Lomé, Lomé, Togo

Submitted: 06-08-2011

Revised: 23-09-2011

Published: 07-04-2012

\begin{abstract}
A B S T R A C T
Background: Plant-based remedies continue to play a key role in the health care of people in Togo; however, there is a lack of published data in medicinal plants and medical practices of the people in the country. Objective: This study was aimed at documenting the plant utilization in the Tem folk medicine in the central region of Togo. Materials and Methods: An ethnobotanical survey was conducted with traditional healers in the central region of Togo using a semistructured questionnaire. Results: This study demonstrated that local specialists in the central region of Togo tend to agree with each other in terms of the plants used to treat diabetes $(\mathrm{ICF}=0.38$ ), infertility, and abdominal pains (ICF $=0.33)$, but cite a much more diverse groups of plants to treat problems related to arterial hypertension, sickle cell disease, and abscess. They use 144 herbal concoctions made of 72 plants, distributed among 36 botanical families. The Euphorbiaceae family with eight species was best represented in terms of the number of species. The species with the highest use value were Khaya senegalensis (Desr.) A. Juss. (Meliaceae) (UV = 0.36), Anthocleista djalonensis A. Chev. (Gentianaceae) (UV = 0.27), Trichilia emetica Vahl (Meliaceae) (UV $=0.25$ ), and Sarcocephalus latifolius (Sm.) E. A. Bruce (Rubiaceae) $(U V=0.21)$. They also rely on the timing in the plant processing and the administration of herbal remedies. Conclusion: All these findings are based on empirical observations; laboratory screenings are needed to check the effectiveness of these plants.
\end{abstract}

Key words: Chronobiology, chronotherapy, folk medicine, herbal concoctions, plant administration, traditional healers

\section{INTRODUCTION}

Plants have formed the basis of traditional medicine (TM) that was used thousands years ago by human beings. Until today plant-based medicine continues to play a key role in the healthcare systems of the developing countries, where modern drugs are not usually affordable. ${ }^{[1,2]}$ Indeed, it has been estimated that up to $80 \%$ people only rely on TM for their primarily health care in Africa. ${ }^{[3]}$ The massive use of plants is encouraged by their efficiency, their availability, and the low cost of herbal concoctions. ${ }^{[4,5]}$ Although many plants have been screened with success for several biological activities, ${ }^{[6-9]}$ some plants have proven very toxic. ${ }^{[10,11]}$ Recently, our team identified some critical points in the plant processing and several risks related to

Address for correspondence:

Dr. Karou D. Simplice, Ecole Supérieure des Techniques

Biologiques et Alimentaires (ESTBA-UL), Université de Lomé,

BP 1515, Lomé, Togo. E-mail: simplicekarou@hotmail.com the ingestion of contaminated herbal concoctions. ${ }^{[12]}$ The adverse effects consecutive to the administration of some plant remedies were also listed although this was only based on the empirical observations of the traditional healers $(\mathrm{TH}) \cdot{ }^{[13]}$

The studies addressing the toxic effects of herbal concoctions have been often focused on the modification of physiological and histological parameters consecutive to the administration of the drugs regardless to the time of administration. ${ }^{[14-16]}$ A few studies have discussed the "timing" factor in the harvest of plant materials, in the preparation, and the administration of the herbal concoctions. ${ }^{[17,18]}$ Yet this factor may play an important role in the diagnosis, treatment, healing, and poisoning associated with taking medication. ${ }^{[19-21]}$ In fact, in humans and in plants, there are regular successions of high and low biological activities during the $24 \mathrm{~h}$ or even during a year. ${ }^{[22]}$ The susceptibility of an organ or a tissue depends on the timing of drug administration. ${ }^{[17,22]}$ In addition, 
the synthesis of enzymes and active principles in plants evolves in a time-dependent manner. ${ }^{[15]}$ For a given drug and for a given organism, there are some moments of less therapeutic effects or toxicity, thus the administration of medicine regardless of the time would expose the patient to undesirable effects. ${ }^{[21,23]}$

The purposes of this study were to document the plant utilization and to evaluate the importance of the "timing" factor in the diagnosis, the harvest of the plant materials and the administration of drugs in the TM of Tem tribe native from the central region of Togo.

\section{MATERIALS AND METHODS}

\section{Study area}

Togo is a western African country lying between the Republic of Burkina Faso in the north, the Republic of Benin in the east, the Republic of Ghana in the west, and the Atlantic Ocean in the south. The country is divided into five economic regions from the north to the south: the Savannah Region, Kara Region, the Central Region, Plateaux Region, and the Maritime Region. The Central Region is located between $0^{\circ} 15^{\prime}-1^{\circ} 35^{\prime}$ north and $8^{\circ}-9^{\circ} 15^{\prime}$ east. It is made up of four prefectures: Tchaoudjo, Tchamaba, Sotouboua, and Blitta [Figure 1], and is bordered to the north by the Kara Region, to the west by the Republic of Ghana, to the East by the Republic of Benin and to the south by the Plateaux Region. It consists of a total area of $13430 \mathrm{sq}$. $\mathrm{km}$ and occupies approximately $23.73 \%$ of the total 56,6000 sq. km. land area of Togo mainland. The region belongs to the tropical zone with one rainy season from April to October and one dry season from October to March. It receives $1200-1500 \mathrm{~mm}$ total rainfall annually. The annual temperatures are between 20 and $32^{\circ} \mathrm{C}$. The vegetation is essentially constituted of tree and bush savannah with excellent biodiversity of medicinal plants. This study was carried out in the Tchaoudjo prefecture. The prefecture is inhabited by 180,400 people, the main ethnic groups being the Tem people. ${ }^{[24]}$ They are mainly muslims; and agriculture and trade are their principal activities.

\section{Data collection}

Direct interviews with $73 \mathrm{TH}$ were performed between March and July 2009 using a semi-structured questionnaire, after their informed consent. Each TH was asked to sign a consent form certifying his/her agreement with the form which was edited to explain the importance of the information they would provide. All TH were members of the Study and Research in Applied Traditional Medicine Centre of the Central Region in Togo (CERMETRA-RC). CERMETRA-RC is a non-governmental organization created in August 2001. The organization involves TH of the four prefectures of the Central Region in Togo. The

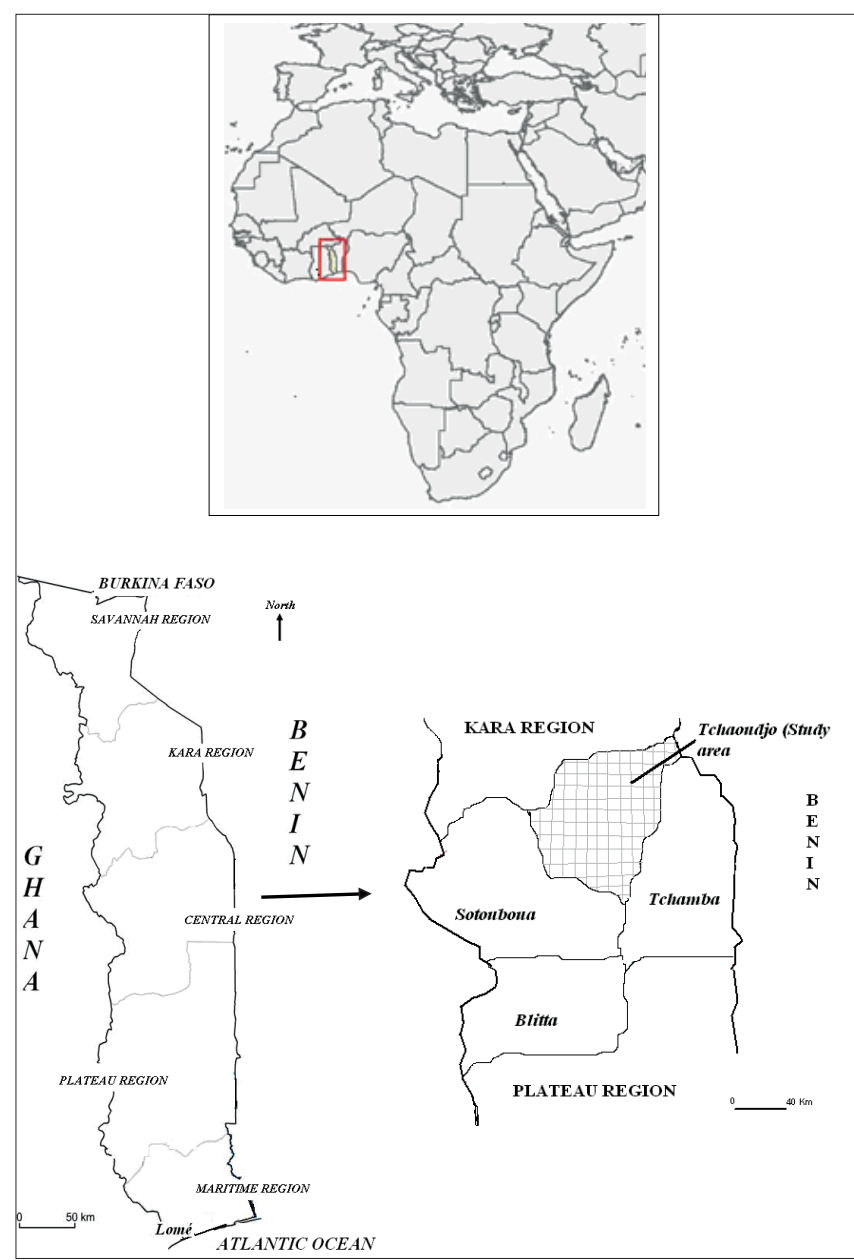

Figure 1: Maps Africa showing Togo, Central Region and the study area

goals of CEMETRA-RC are the training and the counseling of the TH about the management of the patients and the preservation of the environment, principally the protection of vulnerable species used in TM. The organization has a centre where the TH follow up their patients and a botanic garden where they grow desired species. ${ }^{[24]}$ The organization works in collaboration with researchers of University of Lomé. The interviewed TH were from the Tchaoudjo prefecture [Figure 1]. Questions asked were about:

i. TH identity, i.e. name and surname, sex, age;

ii. Educational level;

iii. Origin of the knowledge;

iv. Status of the TH, i.e. full time professional $\mathrm{TH}$ or partially time professional $\mathrm{TH}$;

v. The disease, i.e. name of the disease in the local language;

vi. Remedies, i.e. remedy name, number of plants in the remedy, local names of the plants, used parts, remedy formulation, administration route;

vii Importance of the timing in the diagnosis, plants collection, medicine administration, and therapy. 


\section{Plant identification}

After interviews, preliminary identification of the plants was done in the field by a botanist. Afterwards, herbarium specimens were prepared and photographs were taken to aid in the confirmation of the identity of the plants. Plant identities were confirmed by comparison with available voucher specimens in the Herbarium by Professor Akpagana of the Botany Department, University of Lomé, using taxonomic keys of online databases of the West African Plants Database on the website: http://www. westafricanplants.senckenberg.de/root/index.php?page_ $\mathrm{id}=5$. Nomenclature of species was done using the online data base of the IPNI website: http://www.ipni.org/ipni/ plantnamesearchpage.do.

\section{Data analysis}

An Excel spread sheet was used to make simple calculations and determine plant frequencies. The informant consensus factor (ICF) was calculated for each category to identify the agreements of the informants on the reported cures for diseases. The ICF was calculated by recording the number of use citations in each category (Nuc) minus the number of species used (Ns), and divided by the number of use citations in each category minus one. ${ }^{[25,26]}$

$\mathrm{ICF}=(\mathrm{Nuc}-\mathrm{Ns}) /(\mathrm{Nuc}-1)$

The use value (UV), a quantitative method that demonstrates the relative importance of species known locally, was calculated according to the following formula: ${ }^{[27]}$

$\mathrm{UV}=\Sigma U / n$, where $\mathrm{UV}$ is the use value of a species; $\Sigma U$ is the total number of citations per species, and $n$ the number of informants. These data are helpful in determining the plants with the highest use, most frequently indicated in

\begin{tabular}{|c|c|c|c|c|}
\hline Sex & Males & Females & & \\
\hline Respondents & 61 & 12 & & \\
\hline Percentages & 83.56 & 16.44 & & \\
\hline Ages groups & $20-30$ years & $31-40$ years & $\begin{array}{l}41-50 \\
\text { years }\end{array}$ & $\begin{array}{c}\geq 51 \\
\text { years }\end{array}$ \\
\hline Respondents & 6 & 9 & 21 & 37 \\
\hline Percentages & 8.22 & 12.33 & 28.77 & 50.68 \\
\hline $\begin{array}{l}\text { Educational } \\
\text { level }\end{array}$ & Illiterates & Primary & Secondary & $\begin{array}{c}\text { Uni- } \\
\text { versity }\end{array}$ \\
\hline Respondents & 32 & 27 & 13 & 1 \\
\hline Percentages & 43.83 & 36.99 & 17.81 & 1.37 \\
\hline $\begin{array}{l}\text { Origin of the } \\
\text { knowledge }\end{array}$ & $\begin{array}{l}\text { Familial } \\
\text { heritage } \\
\text { exclusively }\end{array}$ & $\begin{array}{l}\text { Divine } \\
\text { revelation } \\
\text { exclusively }\end{array}$ & $\begin{array}{l}\text { Initiation } \\
\text { from a TH } \\
\text { exclusively }\end{array}$ & Other \\
\hline Respondents & 61 & 7 & 3 & 2 \\
\hline Percentages & 83.56 & 9.59 & 4.11 & 2.74 \\
\hline $\begin{array}{l}\text { Status of the } \\
\mathrm{TH}\end{array}$ & $\begin{array}{l}\text { Full time TH } \\
\text { professional }\end{array}$ & $\begin{array}{l}\text { Partially TH } \\
\text { professional }\end{array}$ & & \\
\hline Respondents & 65 & 8 & & \\
\hline Percentages & 89.04 & 10.96 & & \\
\hline $\begin{array}{l}\text { Religion of } \\
\text { the TH }\end{array}$ & Moslem & Animist & & \\
\hline Respondents & 62 & 11 & & \\
\hline Percentages & 84.93 & 15.07 & & \\
\hline
\end{tabular}

the treatment of an ailment. ${ }^{[28]}$

\section{RESULTS}

\section{Socio-cultural habits of the traditional healers}

A total of 73 TH, 61 men and 12 women were interviewed. The average age of $\mathrm{TH}$ was $53.29 \pm 11.47$ years. The minimum age was 24 years, and the maximum 92 years. All the TH were from the Tem tribe, and all of them speak the local language, "Kotokol", in which interviews were conducted. More than $50 \%$ of the $\mathrm{TH}$ could read and write French. The majority of them $(80 \%)$ claimed that they inherited the medicinal practice from their family. They were mainly farmers but the exercise of the TM was their first occupation. Table 1 displays the detailed socio professional habits of the healers.

\section{Consensus factor among specialists}

The highest ICF values were linked to problems related to diabetes mellitus (0.38), abdominal pains and intestinal parasites (0.33), delivery and female problems (0.31), and male infertility and impotence (0.30). The categories with the lowest ICF value were abscess (0.05), witchcraft and mental illness (0.00), and sickle cell disease (0.00). The number of used species varied considerably among categories. The treatments of abdominal pains and intestinal parasites, malaria and headache, and hemorrhoids required the highest number of species $(55.56 \%, 52.78 \%$, and $43.06 \%$, respectively). A more detailed description of each category is presented in Table 2.

\begin{tabular}{|c|c|c|c|c|c|}
\hline Categories & Species & $\begin{array}{c}\% \text { All } \\
\text { species }\end{array}$ & Citations & $\begin{array}{c}\% \text { All } \\
\text { use } \\
\text { citations }\end{array}$ & ICF \\
\hline $\begin{array}{l}\text { Abdominal } \\
\text { pains and } \\
\text { intestinal } \\
\text { parasites }\end{array}$ & 40 & 55.56 & 59 & 18.50 & 0.33 \\
\hline Hemorrhoids & 31 & 43.06 & 40 & 12.54 & 0.23 \\
\hline $\begin{array}{l}\text { Malaria and } \\
\text { headache }\end{array}$ & 38 & 52.78 & 43 & 13.48 & 0.12 \\
\hline Epilepsy & 22 & 30.56 & 28 & 8.78 & 0.22 \\
\hline $\begin{array}{l}\text { Delivery } \\
\text { and female } \\
\text { problems }\end{array}$ & 19 & 26.39 & 27 & 8.47 & 0.31 \\
\hline Abscess & 21 & 29.17 & 22 & 6.90 & 0.05 \\
\hline $\begin{array}{l}\text { Witchcraft } \\
\text { and mental } \\
\text { illness }\end{array}$ & 28 & 38.89 & 28 & 5.33 & 0 \\
\hline $\begin{array}{l}\text { Sickle cell } \\
\text { disease }\end{array}$ & 14 & 19.44 & 14 & 4.39 & 0 \\
\hline $\begin{array}{l}\text { Male } \\
\text { infertility and } \\
\text { impotence }\end{array}$ & 8 & 11.11 & 11 & 3.45 & 0.30 \\
\hline Hypertension & 10 & 13.89 & 11 & 3.45 & 0.10 \\
\hline Diabetes & 6 & 8.33 & 9 & 2.82 & 0.38 \\
\hline
\end{tabular}




\section{Local specialists and medicinal plants}

This study allowed the identification of 144 herbal concoctions made of 72 plants, distributed among 36 botanical families. The Euphorbiaceae family, with eight species was best represented in terms of the number of species, followed by Fabaceae-Mimosoideae (five species), Fabaceae_Caesalpinioideae, Fabaceae_Faboideae and Meliaceae (four species each). When analyzing the number of citations for the plant parts used to prepare local remedies, a preference for the use of roots (48.21\%), leaves $(30.36 \%)$, and stem bark $(16.67 \%)$ was noticeable [Figure 2]. The use of seeds, fruits, and thorn was less common. With regard to the formulation, the use of powders had the highest relative value $(47.60 \%)$, followed by the use of decoctions (40.87\%). The main route of administration is oral, accounting for $70.55 \%$ remedies. The other routes such as anal, body bath, and direct application on the skin account for less than 10\% each [Figure 2].

Table 3 displays the used species, the use values, and the
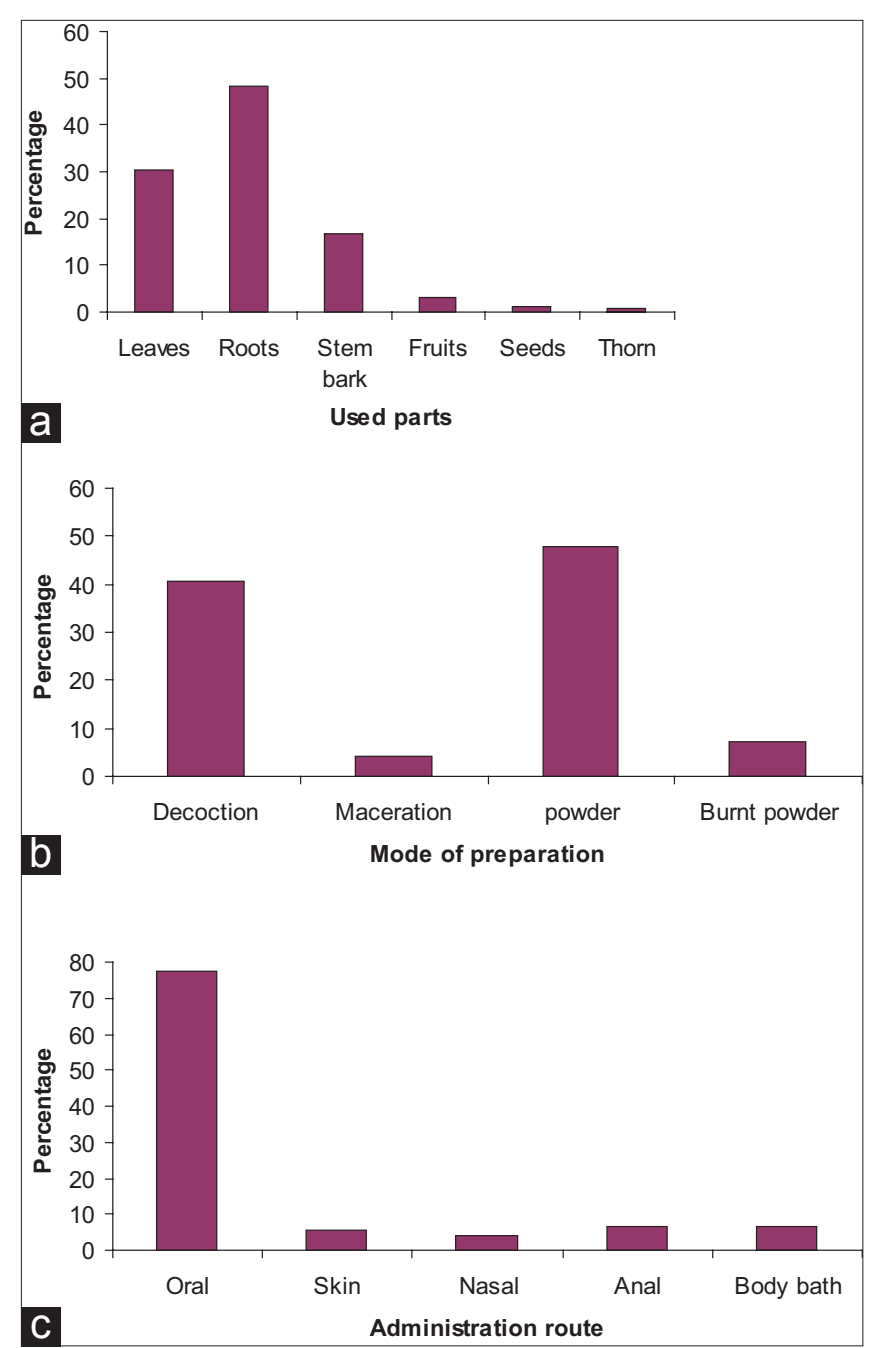

Figure 2: Used parts, mode of preparation and mode of administration of herbal concoctions in the Tem folk medicine mode of administration. The species with the highest use value was Khaya senegalensis (Desr.) A. Juss., local name "Frimou", with an UV value of 0.36 . The main importance of the plant is related to its use in the treatment of hemorrhoids, malaria, sickle cell, stomachache, hypertension, epilepsy, female infertility, and diabetes. The roots, the stem bark, and the leaves of the plant are used in the form of decoction or powder, administrated by anal or oral rout. Anthocleista djalonensis A. Chev., called "Assoubobissaou" had the second highest UV (0.27). Its main medicinal uses are the treatments of hypertension, hemorrhoids, syphilis, female infertility, diabetes, hernia, and male infertility. The roots, the stem bark, and the leaves of the plants are used in concoctions including powders, decoctions, and macerations. The main mode of administration includes the oral, anal, and body bath. Trichilia emetica Vahl, locally known as "Adjindjinkpizou" was ranked third with UV of 0.25 . The plant is used in the treatment of hemorrhoids, mental illness, epilepsy, abscess, typhoid fever, malaria, hypertension, witchcraft, and the trouble of sight. The main used parts are the leaves and the roots in the form of powder, decoction, and maceration. The concoctions are administrated by oral rout or direct application on the skin or by body bath. Sarcocephalus latifolius (Sm.) E. A. Bruce known as "Kidjitchilou" with an UV value of 0.21 was ranked fourth. The plant is used in the treatment of epilepsy, hemorrhoids, sinusitis, sickle cell, witchcraft, female infertility, abscess, abdominal pain, and malaria. The concoctions involved the roots, the fruits, and the leaves of the plants in the form of powder and decoction administrated by direct application on the skin, by oral rout or body bath.

The "timing" factor in the development clinical symptoms of several diseases

According to the interviewed TH, the peak of clinical symptoms varies in the time and according to the disease [Table 4]. The appearance of a repetitive clinical symptom at a particular moment of the day and/or the year may be important to diagnose the disease. Six diseases were cited for this purpose. According to Table 4, the peak of the harmful effects of epilepsy is in the full moon and the occurrence of the symptoms seems to follow a circamensual rhythm. The peak of harmful effects of abscesses and mental illnesses occurs in the middle of the night resting phase, following a circadian rhythm. The same is true for the development of hypertension and asthma with an acrophase at the end of the resting phase at night. A seasonal peak characterizes the evolution of the clinical manifestations of the sickle cell disease.

The "timing" factor in the harvesting of medicinal plants

According to our investigations, for the same plant, the 
Table 3: Medicinal plants and therapeutic indications

\begin{tabular}{|c|c|c|c|c|c|c|c|}
\hline Scientific name & $\begin{array}{l}\text { Voucher } \\
\text { specimen } \\
\text { number }\end{array}$ & Local name & UV & Organs & $\begin{array}{l}\text { Mode of } \\
\text { preparation }\end{array}$ & Route & Indications \\
\hline \multicolumn{8}{|l|}{ AMARANTHACEAE } \\
\hline $\begin{array}{l}\text { Alternanthera pungens } \\
\text { Kunth } \\
\text { ANACARDIACEAE }\end{array}$ & $1815^{*}$ & Sowasowa & 0.03 & $\mathrm{~L}$ & Dec/mac & Oral & Malaria, hemorrhoids \\
\hline Anacardium occidentale L. & $\begin{array}{l}\text { 4692FDS/ } \\
\text { UL }\end{array}$ & Atcha & 0.01 & $\mathrm{R}$ & Pw & Oral & Epilepsy \\
\hline Mangifera indica L. & $\begin{array}{l}\text { 01797TG } \\
\text { Clt/AK }\end{array}$ & Mango & 0.04 & $\begin{array}{l}\mathrm{Sb} \\
\mathrm{L}\end{array}$ & $\begin{array}{l}\text { Dec } \\
\text { Dec }\end{array}$ & $\begin{array}{l}\text { Oral } \\
\text { Oral }\end{array}$ & $\begin{array}{l}\text { Witchcraft, stomachache } \\
\text { Malaria }\end{array}$ \\
\hline \multicolumn{8}{|l|}{ ANNONACEAE } \\
\hline Annona senegalensis Pers. & $\begin{array}{l}2179 F D S / \\
\text { UL }\end{array}$ & Tchoutchoudè & 0.11 & $\mathrm{R}$ & Pw/dec & Oral & $\begin{array}{l}\text { Stomachache, constipation, } \\
\text { ulcer, mental illness, female } \\
\text { infertility }\end{array}$ \\
\hline \multicolumn{8}{|l|}{ ARECACEAE } \\
\hline Borassus aethiopum Mart. & $1830^{*}$ & Kpirou & 0.05 & $\begin{array}{l}\mathrm{R} \\
\mathrm{R} \\
\mathrm{R}\end{array}$ & $\begin{array}{l}\text { Pw } \\
\text { Pw } \\
\text { Mac }\end{array}$ & $\begin{array}{l}\text { Nasal } \\
\text { Oral } \\
\text { Body bath }\end{array}$ & $\begin{array}{l}\text { Headache } \\
\text { Abscess, malaria } \\
\text { Epilepsy }\end{array}$ \\
\hline $\begin{array}{l}\text { Elaeis guineensis Jacq. } \\
\text { ARISTOLOCHIACEAE }\end{array}$ & $\begin{array}{l}\text { 02485TG } \\
\text { Clt/AK }\end{array}$ & Baou & 0.05 & $\mathrm{R}$ & $\mathrm{Pw} / \mathrm{Dec} / \mathrm{BPw}$ & Oral & Epilepsy \\
\hline Aristolochia albida Duch. & $\begin{array}{l}\text { 02006TG } \\
\text { Clt/AK }\end{array}$ & Acadeyog & 0.08 & $\begin{array}{l}R \\
R \\
L \\
L\end{array}$ & $\begin{array}{l}\text { Pw } \\
P w \\
P w \\
\text { Dec }\end{array}$ & $\begin{array}{l}\text { Oral } \\
\text { Skin } \\
\text { Nasal } \\
\text { Oral }\end{array}$ & $\begin{array}{l}\text { Constipation, hypertension } \\
\text { Bone fracture } \\
\text { Headache } \\
\text { Hernia }\end{array}$ \\
\hline \multicolumn{8}{|l|}{ ASTERACEAE } \\
\hline $\begin{array}{l}\text { Acanthospermum hispidum } \\
\text { DC. }\end{array}$ & $\begin{array}{l}\text { 00749TG } \\
\text { Clt/AK }\left(^{*}\right)\end{array}$ & $\begin{array}{l}\text { Kozosogan } \\
\text { sowasowa }\end{array}$ & 0.01 & $\mathrm{~L}$ & $\mathrm{Dec}$ & Oral & Typhoid fever \\
\hline $\begin{array}{l}\text { Chrysanthellum indicum } \\
\text { DC. } \\
\text { BIGNONIACEAE }\end{array}$ & & Tchogbalayo & 0.01 & $\mathrm{~L}$ & $\mathrm{Pw}$ & Oral & Hemorrhoids \\
\hline \multirow[t]{3}{*}{$\begin{array}{l}\text { Kigelia africana (Lam.) } \\
\text { Benth. }\end{array}$} & $1816^{*}$ & Abliou & 0.12 & $\mathrm{Sb}$ & $\mathrm{Pw} / \mathrm{Dec}$ & Oral & $\begin{array}{l}\text { Male infertility, hemorrhoids, } \\
\text { trouble of lactation, witchcraft }\end{array}$ \\
\hline & & & & $\mathrm{L}$ & $\mathrm{Dec}$ & Oral & Hemorrhoids \\
\hline & & & & $\mathrm{R}$ & Pw & Oral & $\begin{array}{l}\text { Hemorrhoids, abscess, female } \\
\text { infertility }\end{array}$ \\
\hline \multirow[t]{2}{*}{$\begin{array}{l}\text { Stereospermum } \\
\text { kunthianum Cham. }\end{array}$} & $1817^{*}$ & Sogbeliya & 0.08 & $\mathrm{R}$ & $\mathrm{Dec} / \mathrm{Pw}$ & Oral & $\begin{array}{l}\text { Stomachache, mental illness, } \\
\text { abscess, male infertility, female } \\
\text { infertility }\end{array}$ \\
\hline & & & & $\mathrm{Sb}$ & Dec & Oral & Female infertility \\
\hline \multicolumn{8}{|l|}{ FABACEAE- } \\
\hline Burkea africana Hook. & $1818^{*}$ & Digbagbati & 0.07 & $\begin{array}{l}\mathrm{R} \\
\mathrm{R} \\
\mathrm{Sb} \\
\mathrm{L}\end{array}$ & $\begin{array}{l}\text { Pw } \\
\text { Pw } \\
\text { Dec } \\
\text { BPw }\end{array}$ & $\begin{array}{l}\text { Skin } \\
\text { Oral } \\
\text { Oral } \\
\text { Oral }\end{array}$ & $\begin{array}{l}\text { Abscess, bone fracture } \\
\text { Sickle cell } \\
\text { Syphilis } \\
\text { Epilepsy }\end{array}$ \\
\hline Cassia occidentalis L. & $1819^{*}$ & Kitchemtchem & 0.05 & $\begin{array}{l}\mathrm{L} \\
\mathrm{L} \\
\mathrm{R}\end{array}$ & $\begin{array}{l}\mathrm{Pw} \\
\mathrm{BPw} \\
\mathrm{Dec} / \mathrm{Pw}\end{array}$ & $\begin{array}{l}\text { Oral } \\
\text { Oral } \\
\text { Oral }\end{array}$ & $\begin{array}{l}\text { Constipation } \\
\text { Malaria } \\
\text { Epilepsy, abscess }\end{array}$ \\
\hline $\begin{array}{l}\text { Erythrophleum africanum } \\
\text { (Welw. ex Benth.) Harms }\end{array}$ & $1820^{*}$ & Kékéou & 0.03 & $\begin{array}{l}\mathrm{L} \\
\mathrm{R}\end{array}$ & $\begin{array}{l}\mathrm{Pw} \\
\mathrm{Pw}\end{array}$ & $\begin{array}{l}\text { Skin } \\
\text { Oral }\end{array}$ & $\begin{array}{l}\text { Snakebite } \\
\text { Hemorrhoids }\end{array}$ \\
\hline $\begin{array}{l}\text { Piliostigma thonningii } \\
\text { (Schumach.) Milne-Redh. }\end{array}$ & $\begin{array}{l}0469 \text { FDS/ } \\
\text { UL }\end{array}$ & Baco & 0.14 & $\begin{array}{l}\mathrm{R} \\
\mathrm{R}\end{array}$ & $\begin{array}{l}\mathrm{Pw} \\
\mathrm{Pw} / \mathrm{Dec}\end{array}$ & $\begin{array}{l}\text { Anal } \\
\text { Oral }\end{array}$ & $\begin{array}{l}\text { Hemorrhoids } \\
\text { Mental illness sinusitis, fibroids, } \\
\text { intestinal parasites, witchcraft }\end{array}$ \\
\hline CARICACEAE & & & & $\mathrm{Sb}$ & $\mathrm{Dec} / \mathrm{Pw}$ & Oral & Malaria, epilepsy \\
\hline Carica papaya L. & $\begin{array}{l}\text { 00340TG } \\
\text { Clt/AK }\end{array}$ & Borofoudé & 0.11 & $\begin{array}{l}\mathrm{R} \\
\mathrm{Fr} \\
\mathrm{Sd} \\
\mathrm{Sd} \\
\mathrm{L} \\
\mathrm{L}\end{array}$ & $\begin{array}{l}\text { Pw/Dec } \\
\text { BPw } \\
\text { Dec } \\
\text { BPw } \\
\text { Dec } \\
\text { Dec }\end{array}$ & $\begin{array}{l}\text { Oral } \\
\text { Oral } \\
\text { Oral } \\
\text { Oral } \\
\text { Body bath } \\
\text { Anal }\end{array}$ & $\begin{array}{l}\text { Constipation, witchcraft } \\
\text { Vaginal discharge } \\
\text { Intestinal parasites } \\
\text { Witchcraft } \\
\text { Malaria } \\
\text { Trouble of delivery }\end{array}$ \\
\hline
\end{tabular}


Table 3 contd...

\begin{tabular}{|c|c|c|c|c|c|c|c|}
\hline Scientific name & $\begin{array}{l}\text { Voucher } \\
\text { specimen } \\
\text { number }\end{array}$ & Local name & UV & Organs & $\begin{array}{l}\text { Mode of } \\
\text { preparation }\end{array}$ & Route & Indications \\
\hline \multicolumn{8}{|l|}{ CELASTRACEAE } \\
\hline \multirow[t]{4}{*}{$\begin{array}{l}\text { Gymnosporia senegalensis } \\
\text { (Lam.) Loes. }\end{array}$} & \multirow[t]{4}{*}{$\begin{array}{l}3738 \text { FDS/ } \\
\text { UL }\end{array}$} & \multirow[t]{4}{*}{ Tchintchingan } & \multirow[t]{4}{*}{0.14} & $\mathrm{R}$ & $\mathrm{Dec} / \mathrm{Pw}$ & Oral & $\begin{array}{l}\text { Epilepsy, sickle cell, abscess, } \\
\text { stomachache, sexual weakness }\end{array}$ \\
\hline & & & & $\mathrm{R}$ & $\mathrm{BPw}$ & Oral & Malaria \\
\hline & & & & $\mathrm{L}$ & Pw/Dec & Oral & Typhoid fever, hernia \\
\hline & & & & $\mathrm{Sb}$ & Dec & Oral & Male infertility, hemorrhoids \\
\hline \multicolumn{8}{|l|}{ CHRYSOBALANACEAE } \\
\hline Parinari curatellifolia & \multirow[t]{2}{*}{$1821^{*}$} & \multirow[t]{4}{*}{ Millimilou } & \multirow[t]{2}{*}{0.05} & $\mathrm{R}$ & $\mathrm{Pw}$ & Oral & Mental illness \\
\hline Planch. ex Benth. & & & & $\mathrm{Sb}$ & Dec & Oral & Witchcraft \\
\hline \multirow{2}{*}{$\begin{array}{l}\text { Parinari senegalensis Perr. } \\
\text { ex DC. } \\
\text { COMBRETACEAE }\end{array}$} & \multirow[t]{2}{*}{$1822^{*}$} & & \multirow[t]{2}{*}{0.03} & $\mathrm{Sb}$ & Dec & Oral & Syphilis \\
\hline & & & & $\mathrm{R}$ & Pw & Oral & Hemorrhoids \\
\hline \multirow{3}{*}{$\begin{array}{l}\text { Pteleopsis suberosa Engl. } \\
\text { and Diels }\end{array}$} & \multirow{3}{*}{$\begin{array}{l}8078 \text { FDS/ } \\
\text { UL }\end{array}$} & \multirow[t]{3}{*}{ Sisinon } & \multirow[t]{3}{*}{0.05} & $\mathrm{R}$ & $\mathrm{Dec} / \mathrm{Pw}$ & Oral & Sickle cell, hemorrhoids \\
\hline & & & & $\mathrm{Sb}$ & $\mathrm{Pw}$ & Oral & Hypertension \\
\hline & & & & $\mathrm{L}$ & Dec & Oral & Stomachache \\
\hline \multicolumn{8}{|l|}{ Guill. and Perr. } \\
\hline \multicolumn{8}{|l|}{ CUCURBITACEAE } \\
\hline \multirow[t]{3}{*}{ Momordica charantia L. } & 6182 FDS/ & Katchalayo & 0.04 & $\mathrm{~L}$ & $\mathrm{Pw}$ & Oral & Hemorrhoids \\
\hline & UL & & & $L$ & $\mathrm{Dec}$ & Anal & Trouble of delivery \\
\hline & & & & $\mathrm{R}$ & $\mathrm{Pw}$ & Oral & Hemorrhoids \\
\hline EUPHORBIACEAE & & & & & & & \\
\hline Bridelia ferruginea Benth. & $\begin{array}{l}7382 \text { FDS/ } \\
\text { UL }\end{array}$ & Kolou & 0.07 & $\mathrm{R}$ & $\mathrm{Pw} / \mathrm{Dec}$ & Oral & $\begin{array}{l}\text { Malaria, sickle cell, typhoid } \\
\text { fever }\end{array}$ \\
\hline & & & & $\mathrm{Sb}$ & Pw & Oral & Stomachache \\
\hline Euphorbia hirta L. & $1824^{*}$ & Kovoyoyilim & 0.04 & $\mathrm{R}$ & $\mathrm{Dec}$ & Oral & $\begin{array}{l}\text { Female infertility, intestinal } \\
\text { parasites }\end{array}$ \\
\hline & & & & $\mathrm{L}$ & $\mathrm{Pw}$ & Oral & Malaria \\
\hline Jatropha curcas L. & $1825^{*}$ & Sawou & 0.05 & $\mathrm{R}$ & $\mathrm{Dec} / \mathrm{Pw}$ & Anal & Hemorrhoids, stomachache \\
\hline & & kifouloumou & & $\mathrm{L}$ & Mac & Anal & Female infertility \\
\hline Jatropha gossypiifolia L. & CNE & Sawou & 0.04 & $\mathrm{R}$ & $\mathrm{Pw}$ & Anal & Hemorrhoids, stomachache \\
\hline & & kissèmou & & $\mathrm{L}$ & Dec & Oral & Malaria \\
\hline Phyllanthus amarus & 571 FDS/ & Sénisèniyo & 0.04 & $\mathrm{R}$ & $\mathrm{Dec} / \mathrm{Pw}$ & Oral & Stomachache, abscess, \\
\hline Schumach. and Thonn. & & & & $\mathrm{R}$ & BPw & Oral & $\begin{array}{l}\text { malaria, hemorrhoids } \\
\text { Witchcraft }\end{array}$ \\
\hline & & & & $\mathrm{L}$ & Pw & Oral & Hemorrhoids \\
\hline $\begin{array}{l}\text { Phyllanthus muellerianus } \\
\text { (Kuntze) Exell }\end{array}$ & $1826^{*}$ & Librélibré & 0.07 & $\mathrm{R}$ & Pw & Oral & $\begin{array}{l}\text { Epilepsy, stomachache, } \\
\text { headache }\end{array}$ \\
\hline Excoecaria grahamii Stapf & $1827^{*}$ & Katchikadou & 0.11 & $\mathrm{R}$ & $\mathrm{Dec} / \mathrm{Pw}$ & Oral & $\begin{array}{l}\text { Mental illness, female infertility, } \\
\text { stomachache, trouble of } \\
\text { lactation, yellow fever, snakebite }\end{array}$ \\
\hline & & & & $\mathrm{Sb}$ & $\mathrm{Dec}$ & Oral & malaria \\
\hline Flueggea virosa (Roxb. ex & 008 FDS/ & Tchacatchaca & 0.11 & $\mathrm{R}$ & $\mathrm{Pw} / \mathrm{Dec}$ & Oral & Hemorrhoids, stomachache, \\
\hline Willd.) Voigt & UL & & & & & & $\begin{array}{l}\text { sickle cell, epilepsy, diabetes, } \\
\text { snakebite }\end{array}$ \\
\hline & & & & $\mathrm{Sb}$ & Dec & Oral & Diabetes \\
\hline FABACEAE-FABOIDEAE & & & & & & & \\
\hline Philenoptera cyanescens & 4409 FDS/ & Tchèlè & 0.05 & $\mathrm{Sb}$ & Dec & Oral & Female infertility, hypertension \\
\hline (Schumach. and Thonn.) & UL & & 0.01 & $\mathrm{~L}$ & Dec & Body bath & Malaria \\
\hline Roberty & & & & $\mathrm{R}$ & $\mathrm{Pw}$ & Oral & Epilepsy \\
\hline $\begin{array}{l}\text { Millettia thonningii } \\
\text { (Schumach. and Thonn.) } \\
\text { Baker }\end{array}$ & 10822 & Kodoliya & 0.19 & $\mathrm{R}$ & $\mathrm{Pw} / \mathrm{Dec}$ & Oral & $\begin{array}{l}\text { Ulcer, epilepsy, stomachache, } \\
\text { intestinal parasites, } \\
\text { hemorrhoids, diabetes }\end{array}$ \\
\hline & & & & $\mathrm{R}$ & $\mathrm{Pw}$ & Nasal & Headache \\
\hline & & & & $\mathrm{R}$ & $\mathrm{Pw}$ & Skin & Abscess \\
\hline & & & & $\mathrm{Sb}$ & $\mathrm{Dec} / \mathrm{Pw}$ & Oral & Epilepsy, male infertility \\
\hline Pterocarpus erinaceus Poir. & 15 FDS/UL & Tem & 0.03 & $\mathrm{R}$ & $\mathrm{Pw}$ & Oral & Typhoid fever \\
\hline & & & & $\mathrm{Sb}$ & $\mathrm{Dec}$ & Oral & Hemorrhoids \\
\hline Xeroderris stuhlmannii & 10571 FDS/ & Tchalawari & 0.05 & $\mathrm{Sb}$ & $\mathrm{Dec} / \mathrm{Pw}$ & Oral & Witchcraft, fibroids \\
\hline (Taub.) Mendonça and E.P. & UL & & & $\mathrm{R}$ & $\mathrm{Pw}$ & Oral & Typhoid fever \\
\hline Sousa & & & & $\mathrm{R}$ & Pw & Oral & Epilepsy \\
\hline LAMIACEAE & & & & & & & \\
\hline Hyptis suaveolens Poit. & 04184TG & Botifadini & 0.05 & $\mathrm{R}$ & Dec & Oral & Abscess \\
\hline & $\mathrm{Clt} / \mathrm{AK}$ & & & $\mathrm{L}$ & $\mathrm{Dec}$ & $\begin{array}{l}\text { Oral/body } \\
\text { bath }\end{array}$ & Malaria \\
\hline
\end{tabular}


Table 3 contd..

\begin{tabular}{|c|c|c|c|c|c|c|c|}
\hline Scientific name & $\begin{array}{l}\text { Voucher } \\
\text { specimen } \\
\text { number }\end{array}$ & Local name & UV & Organs & $\begin{array}{l}\text { Mode of } \\
\text { preparation }\end{array}$ & Route & Indications \\
\hline \multirow[t]{2}{*}{ Ocimum americanum $\mathrm{L}$. } & 04199TG & Kozosogan & 0.01 & $\mathrm{~L}$ & $\mathrm{PW}$ & Oral & Hemorrhoids \\
\hline & Clt/AK & & & $\mathrm{L}$ & $\mathrm{BPw}$ & Oral & Malaria \\
\hline \multirow[t]{4}{*}{ Ocimum gratissimum L. } & 3892 FDS/ & Kounozorou & 0.08 & $\mathrm{~L}$ & Mac & Anal & Female infertility \\
\hline & UL & & & $\mathrm{L}$ & Pw & Nasal & Headache \\
\hline & & & & $\mathrm{L}$ & $\mathrm{BPw}$ & Oral & malaria \\
\hline & & & & $\mathrm{R}$ & $\mathrm{Pw}$ & Oral & Trouble of lactation, constipation \\
\hline \multicolumn{8}{|l|}{ LILLIACEAE } \\
\hline Aloe vera (L.) Burm.f. & 10857 & Faradjo & 0.01 & $\mathrm{R}$ & Dec & Oral & Diabetes \\
\hline Allium sativum $\mathrm{L}$. & 10856 & Ayo & 0.03 & $\mathrm{R}$ & Dec & Oral & Hemorrhoids \\
\hline \multicolumn{8}{|l|}{ GENTIANACEAE } \\
\hline \multirow{6}{*}{$\begin{array}{l}\text { Anthocleista djalonensis A. } \\
\text { Chev. }\end{array}$} & 2326 FDS/ & Assoubobiss- & 0.27 & $\mathrm{Sb}$ & $\mathrm{Pw} / \mathrm{Dec}$ & Oral & Hypertension, hemorrhoids, \\
\hline & UL & aou & & & & & $\begin{array}{l}\text { syphilis, female infertility, } \\
\text { diabetes }\end{array}$ \\
\hline & & & & $\mathrm{L}$ & Dec & Oral & $\begin{array}{l}\text { Hemorrhoids, malaria, hernia, } \\
\text { stomachache }\end{array}$ \\
\hline & & & & $\mathrm{L}$ & Mac & $\begin{array}{l}\text { Anal/body } \\
\text { bath }\end{array}$ & Female infertility, epilepsy \\
\hline & & & & $\mathrm{R}$ & $\mathrm{Dec} / \mathrm{Pw}$ & Oral & $\begin{array}{l}\text { Hemorrhoids, hypertension, } \\
\text { diabetes, male infertility, female } \\
\text { infertility }\end{array}$ \\
\hline & & & & $\mathrm{R}$ & $\mathrm{BPw}$ & Oral & Epilepsy \\
\hline \multicolumn{8}{|l|}{ STRYCHNACEAE } \\
\hline Strychnos spinosa Lam. & $\begin{array}{l}10779 \text { FDS/ } \\
\text { UL }\end{array}$ & Kpogbovouro & 0.07 & $\mathrm{R}$ & $\mathrm{Pw}$ & Oral & $\begin{array}{l}\text { Witchcraft, stomachache, } \\
\text { headache, mental illness }\end{array}$ \\
\hline \multicolumn{8}{|l|}{ MELIACEAE } \\
\hline \multirow[t]{2}{*}{ Azadirachta indica A. Juss. } & $\begin{array}{l}\text { 04647Tg } \\
\text { Clt/AK }\end{array}$ & Tiyo & 0.07 & $\mathrm{R}$ & $\mathrm{Pw}$ & Oral & $\begin{array}{l}\text { Malaria, hemorrhoids, } \\
\text { constipation }\end{array}$ \\
\hline & & & & $\mathrm{L}$ & Dec & Oral & Malaria \\
\hline \multirow{4}{*}{$\begin{array}{l}\text { Khaya senegalensis (Desr.) } \\
\text { A.Juss. }\end{array}$} & 10641 FDS/ & Frimou & 0.36 & $\mathrm{R}$ & $\mathrm{PW}$ & Anal & Hemorrhoids \\
\hline & UL bis & & & $\mathrm{R}$ & $\mathrm{Pw} / \mathrm{Dec}$ & Oral & $\begin{array}{l}\text { Hemorrhoids, malaria, } \\
\text { sickle cell, stomachache, } \\
\text { hypertension, fainting }\end{array}$ \\
\hline & & & & $\mathrm{Sb}$ & $\mathrm{Pw} / \mathrm{Dec}$ & Oral & $\begin{array}{l}\text { Hemorrhoids, stomachache, } \\
\text { epilepsy, female infertility, } \\
\text { diabetes }\end{array}$ \\
\hline & & & & $\mathrm{L}$ & $\mathrm{Pw}$ & Oral & Fainting \\
\hline \multirow[t]{3}{*}{$\begin{array}{l}\text { Pseudocedrela kotschyi } \\
\text { (Schweinf.) Harms }\end{array}$} & $\begin{array}{l}7719 \text { FDS/ } \\
\text { UL }\end{array}$ & Ditotorè & 0.12 & $\mathrm{R}$ & Pw/Dec & Oral & $\begin{array}{l}\text { Epilepsy, mental illness, } \\
\text { abscess, malaria, hemorrhoids }\end{array}$ \\
\hline & & & & $\mathrm{L}$ & $\mathrm{BPw}$ & Oral & Epilepsy \\
\hline & & & & $\mathrm{L}$ & Dec & Oral & Stomachache \\
\hline \multirow[t]{4}{*}{ Trichilia emetica Vahl } & $\begin{array}{l}308 \text { FDS/ } \\
\text { UL }\end{array}$ & $\begin{array}{l}\text { Adjindjinkpi- } \\
\text { zou }\end{array}$ & 0.25 & $\mathrm{R}$ & $\mathrm{Pw} / \mathrm{Dec}$ & Oral & $\begin{array}{l}\text { Hemorrhoids, mental illness, } \\
\text { epilepsy, abscess, typhoid fever, } \\
\text { malaria, hypertension }\end{array}$ \\
\hline & & & & $\mathrm{R}$ & $\mathrm{BPw}$ & Oral & Witchcraft, epilepsy \\
\hline & & & & $\mathrm{R} / \mathrm{L}$ & $\mathrm{Pw}$ & Skin & Abscess \\
\hline & & & & $\mathrm{L}$ & Mac & Body bath & Trouble of sight \\
\hline \multicolumn{8}{|l|}{ FABACEAE- } \\
\hline \multicolumn{8}{|l|}{ MIMOSOIDEAE } \\
\hline Dichrostachys cinerea (L.) & 299 FDS/ & Bouvoun & 0.03 & Th & Burnt Pw & Oral & Witchcraft \\
\hline Wight and Arn. & UL & & & & & & \\
\hline Entada africana Guill. and & $1828 *$ & N'doulou & 0.05 & $\mathrm{R}$ & $\mathrm{Pw}$ & Oral & Constipation, female infertility \\
\hline \multirow[t]{2}{*}{ Perr. } & & & & $\mathrm{L}$ & $\mathrm{Pw}$ & Oral & Constipation \\
\hline & & & & $\mathrm{Sb}$ & $\mathrm{Pw}$ & Oral & Sickle cell, trouble of lactation \\
\hline Parkia biglobosa (Jacq.) R. & 9468 FDS/ & Soulou & 0.10 & $\mathrm{R}$ & Pw & Skin & Abscess \\
\hline \multirow[t]{3}{*}{ Br. ex G. Don } & UL & & & $\mathrm{R}$ & $\mathrm{Pw} / \mathrm{Dec}$ & Oral & Stomachache, epilepsy \\
\hline & & & & $\mathrm{Sb}$ & Dec & Oral & Epilepsy \\
\hline & & & & $\mathrm{L}$ & Pw & Oral & Ulcer, headache \\
\hline \multirow{4}{*}{$\begin{array}{l}\text { Pericopsis laxiflora (Benth.) } \\
\text { Meeuwen }\end{array}$} & 2324 FDS/ & Tchèmany & 0.12 & $\mathrm{R}$ & $\mathrm{Pw} / \mathrm{Dec}$ & Oral & Stomachache, headache, \\
\hline & UL & & & & & & $\begin{array}{l}\text { yellow fever, intestinal parasites, } \\
\text { Sickle cell, hemorrhoids, } \\
\text { abscess }\end{array}$ \\
\hline & & & & $\mathrm{R}$ & Mac & Body bath & Epilepsy \\
\hline & & & & $\mathrm{Sb}$ & Dec & Oral & Malaria \\
\hline
\end{tabular}


Table 3 contd...

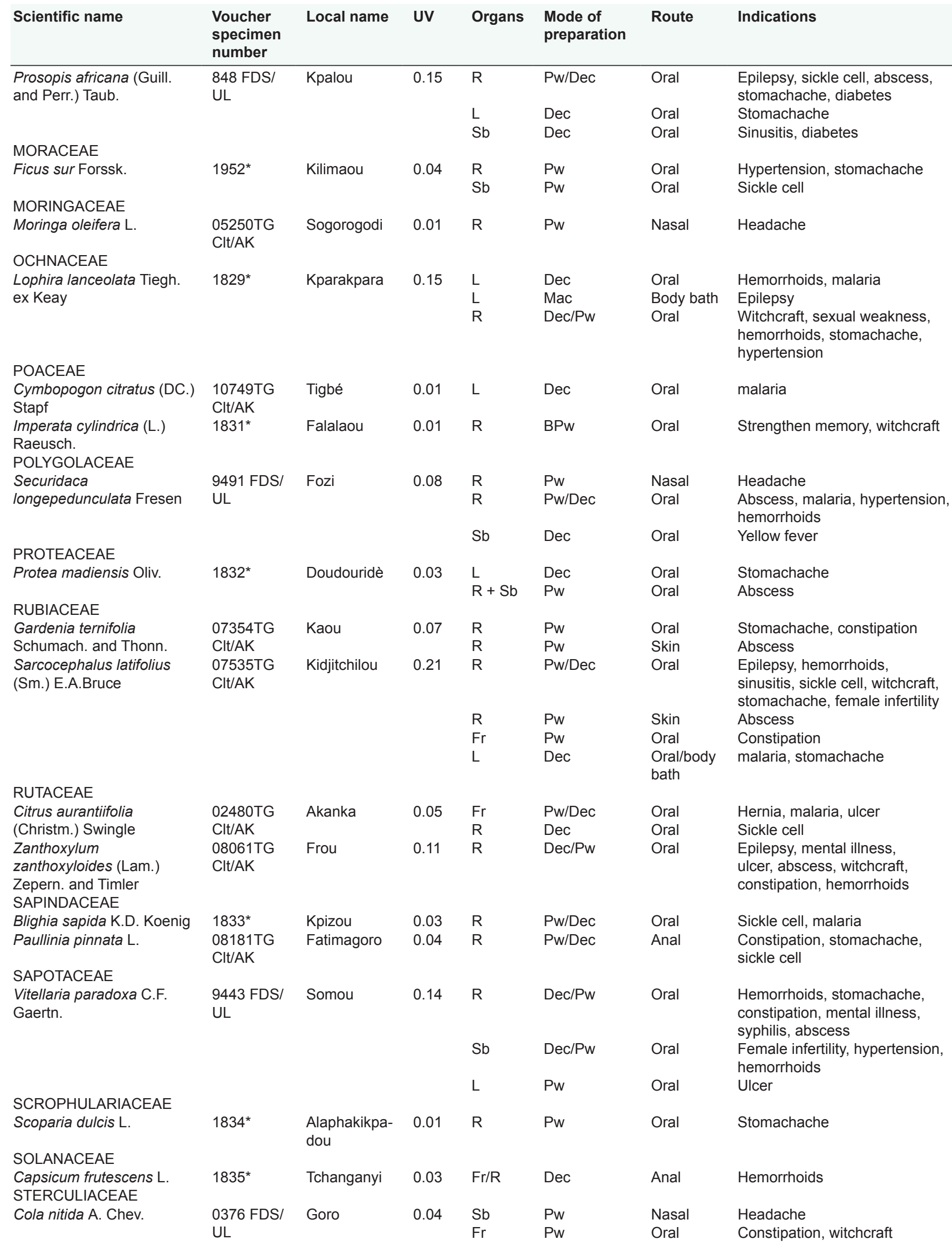


Table 3 contd..

\begin{tabular}{|c|c|c|c|c|c|c|c|}
\hline Scientific name & $\begin{array}{l}\text { Voucher } \\
\text { specimen } \\
\text { number }\end{array}$ & Local name & UV & Organs & $\begin{array}{l}\text { Mode of } \\
\text { preparation }\end{array}$ & Route & Indications \\
\hline \multicolumn{8}{|l|}{ STRYCHNACEAE } \\
\hline Strychnos spinosa Lam. & $\begin{array}{l}10779 \text { FDS/ } \\
\text { UL }\end{array}$ & Kpogbovouro & 0.07 & $\mathrm{R}$ & Pw & Oral & $\begin{array}{l}\text { Witchcraft, stomachache, } \\
\text { headache, mental illness }\end{array}$ \\
\hline \multicolumn{8}{|l|}{ TILIACEAE } \\
\hline \multirow[t]{2}{*}{ Grewia mollis Juss. } & $1836^{*}$ & Bolo & 0.03 & $\mathrm{~L}$ & Pw & Oral & Mental illness \\
\hline & & & & $\mathrm{Sb}$ & Pw & Skin & Abscess \\
\hline \multicolumn{8}{|l|}{ VERBENACEAE } \\
\hline Premna quadrifolia & $1837^{*}$ & Tchakpeya & 0.03 & $\mathrm{R}$ & Pw & Oral & Female infertility \\
\hline Schumach. and Thonn. & & & & $\mathrm{L}$ & $\mathrm{BPw}$ & Oral & Epilepsy \\
\hline $\begin{array}{l}\text { Stachytarpheta indica (L.) } \\
\text { Vahl }\end{array}$ & $\begin{array}{l}820 \text { FDS/ } \\
\text { UL }\end{array}$ & $\begin{array}{l}\text { Tchouboulou- } \\
\text { zou }\end{array}$ & 0.01 & $\mathrm{R}$ & Mac & Body bath & Trouble of sight \\
\hline \multirow[t]{2}{*}{ Tectona grandis L.f. } & 09267TG & Tantouna & 0.04 & $\mathrm{~L}$ & Dec & Oral & Anemia \\
\hline & Clt/AK & & & $\mathrm{L}$ & $\mathrm{Dec}$ & $\begin{array}{l}\text { Oral/body } \\
\text { bath }\end{array}$ & malaria \\
\hline \multicolumn{8}{|l|}{ VITACEAE } \\
\hline $\begin{array}{l}\text { Cissus aralioides (Baker) } \\
\text { Planch. }\end{array}$ & $1838^{*}$ & Bodi & 0.05 & $\mathrm{R}$ & Pw/Dec & Oral & $\begin{array}{l}\text { Intestinal parasites, malaria, } \\
\text { stomachache }\end{array}$ \\
\hline
\end{tabular}

L: leaves, Fr: fruits, R: roots, Sb: stem bark, S: seeds, Pw: powder, Dec, decoction, Mac: maceration, BPw, burnt powder, Th, thorn

\begin{tabular}{llc}
\multicolumn{3}{l}{$\begin{array}{l}\text { Table 4: Periodicity of symptoms of several } \\
\text { diseases }\end{array}$} \\
\hline Diseases & Peak of symptoms & Number of TH \\
\hline Epilepsy & Full moon & $18 / 73$ \\
& $\left(10^{\text {th }}\right.$ to $15^{\text {th }}$ days of the month) & \\
Abscess & Night & $14 / 73$ \\
Asthma & Early morning & $19 / 73$ \\
Sickle cell & Cold time & $21 / 73$ \\
& (November to February) & \\
Arterial & Early morning & $23 / 73$ \\
hypertension & & \\
$\begin{array}{l}\text { Mental } \\
\text { illness }\end{array}$ & Midnight & $09 / 73$ \\
\hline
\end{tabular}

time of harvest can vary from one organ to another. Generally, the roots and the stem bark are harvested during the dry season and the leaves during the rainy season, most frequently in the morning. In this study, 29 plants (40.28\%) must be harvested at specific moment of the day and/ or the year to be efficient. These plants are incorporated in the preparation of 19 medical recipes used to cure the following diseases: headache, hemorrhoids, constipation, mental illness, female infertility, epilepsy, stomach pain, sickle cell anemia, malaria, fainting, and hypertension. As an example, the leaves of Khaya senegalensis are harvested in the morning in the rainy season. These leaves are pulverized to give a recipe called "Iralèda" taken orally in the case of fainting. Trichilia emetica leaves are harvested in the night during the dry season, and they are used in the form of decoction orally against overwork. Sarcocephalus latifolius roots are harvested in the dry season in the evening. The powder is incorporated in a recipe, "Gnon" used in the treatment of stomach pains. On the other hand, the leaves are harvested in the morning in the dry season and are used in the form of decoction in association with the roots of Bridelia ferruginea and Cissus aralioides against malaria. This recipe is traditionally known as "Lakaza."

The "timing" factor in the administration of herbal medicines

According to the 73 healers interviewed in this survey, the cure or the occurrence of adverse reactions after ingestion of a drug depends on the time of administration. A total of 72 recipes were identified for this purpose. These recipes prepared with 60 plants have their optimal therapeutic effects either in the morning, midday, and/or evening. For example, 11 recipes made from 22 plants were identified as having an optimal activity if administered in the morning. These recipes were mostly composed of roots, and presented in the form of powder and administered orally. "Lizi", for example, is a concoction made of Carica papaya seeds and roots of Euphorbia birta. This decoction is administered orally to treat intestinal parasites. Its activity is optimal if it is taken in the morning. Similarly, maceration of leaves of Alternantera pungens, known as the "Sinama" and used orally to treat malaria, has an optimal activity when taken in the morning. Nineteen recipes made of 37 plants were recorded as having optimal activity at night. These recipes are mostly from roots and used as powder or decoction orally. "Maza", made of powdered roots of Khaya senegalensis and Piliostigma thonningii, is presented in the form of capsule. It is administered anally to treat hemorrhoids. Its activity is optimal in the evening. Similarly "Labitala", a decoction of the roots of Excoecaria grahamii and Euphorbia birta, is preferably administered orally at night to treat female infertility.

The time of administration and the occurrence of adverse events

The survey showed that 44 recipes from 56 plants could be a source of toxic or undesirable effects if the time of 
administration is not respected. Thus, we identified eight recipes from 16 plants that have acute toxicities sometimes if taken in the morning. For example, the bark Lophira lanceolata is used in the form of a powder called "Essovalè" administered orally in the case of female infertility. Administered in the morning, the recipe may cause abdominal pain or itching. Similarly, the roots of Aristolochia albida are used in the form of powder against cough. Oral administration in the morning can cause diarrhea and abdominal pain. Four recipes based on 11 plants have their peak of toxicity in the evening. "N'Ketekina" is a powder made of the fruits of Sracopcephalus latifolius and the roots of Entada africana. This recipe is used orally against constipation, but administered in the evening it can cause diarrhea, vomiting, and general weakness.

\section{DISCUSSION}

The main objective of this study was to investigate the use of the plants in the Tem TM. Our results revealed that the interviewed TH were mostly senior male citizens entirely dedicated to the exercise of TM. The low number of illiterates is due to the fact that these TH are members of the CERMETRA. In fact the institution requires a minimum educational level. By adhering to CERMETRA, the $\mathrm{TH}$ who are illiterate receive courses to learn speaking and reading French and the local language. ${ }^{[13]}$

There seems to be a tendency for a few plant families to stand out in any pharmacopoeia. In a survey on antimalarial plants conducted in the Maritime Region of Togo, Koudouvo et al. ${ }^{[29]}$ identified 52 plants species belonging to 29 families, the most represented being the Rubiaceae and Rutaceae. In the study conducted in the Central Plate of Burkina Faso by Nadembega et al. ${ }^{[30]}$ the families Caesalpiniaceae, Poaceae, Mimosaceae and Fabaceae were classified as the richest in species citations. Maroyi et al. ${ }^{[31]}$ recorded 61 plant species as useful in traditionally curing of various human diseases in the Nhema communal area in Zimbabwe. These medicinal plants were distributed among 28 families, the largest proportion belonging to Fabaceae and Anacardiaceae families. Telefo $e t$ al..$^{[32]}$ also identified 46 plant species belonging to 26 families, the largest number of species being recorded in the Asteraceae and Acanthaceae. In this study, the largest number of species belonged to the Euphorbiaceae family. Preference for their use may be related as much to their ready availability, for they are common in this area, as to factors related to their biological activity.

Khaya senegalensis was the plant with highest UV. The main therapeutic indications of this plant were: hemorrhoids, malaria, sickle cell, stomachache, hypertension, female infertility, and diabetes. In fact this plant is well known by TH native from western Africa, and it assumed to cure several diseases. In Burkina Faso, the plant is used in the treatment of malaria and stomachache. ${ }^{[33]}$ In Ivory Coast, the bark of the plant is used in themanagement of externalandinternalwounds, diarrhea, and dysentery. ${ }^{[34]}$ In Mali, in addition to the treatment of wounds, the plant is also used in the management of snake or insect bites. ${ }^{[3]}$ In Guinea, the plant is used to treat infectious diseases including sexually transmitted ones. ${ }^{[36]}$ Indeed, there are some similarities in the use of Khaya senegalensis in Western Africa, the main indication being the treatment of microbial infections. Following theses indications, laboratory screenings were conducted on the plant. Karou et al. ${ }^{[37,38]}$ found weak antiplasmodial and antibacterial activities of the crude extracts of leaves and bark of the plant. The phytochemical studies on the plant resulted in the isolation of several compounds, the most commons being the limonoids with antimicrobial activity. ${ }^{[39]}$ Anthocleista djalonensis is used in the management of hypertension, hemorrhoids, syphilis, female infertility, diabetes, malaria, hernia, and stomachache. Gbolade ${ }^{[40]}$ also found similar usage of the plant in Nigeria. The in vitro screening of the plant revealed an interesting antimicrobial activity of the crude extract. ${ }^{[11,42]}$ There is a grate similarity in the use of Sarcocephalus latifolius and Trichilia emetica in TM in West Africa. ${ }^{[37,43]}$ Of the two plants, Sarcocephalus latifolius previously known as Nauclea latifolia is well investigated for several biological activities including antimalarial, antidiabetic, and antimicrobial properties. ${ }^{[44]}$

Overall our results indicated that the TM in Togo shares similarities with the TM of the subregion in the use of plants. We also investigated the importance of timing in the Togolese folk medicine. In this study, the 73 respondents claimed they rely on time in their daily practice. We first checked the knowledge of $\mathrm{TH}$ on the periodicity of some clinical symptoms of several diseases. The surveyed TH indicated that the periodicity of the clinical symptoms was circamensual for epilepsy, circadian for the skin abscesses, certain mental disorders, hypertension and asthma, and seasonal for the sickle cell anemia. These data are consistent with the literature. Indeed, the peak of the abnormal electrical discharge in the brain that is the cause of epilepsy appears at the full moon. The pain of skin abscesses can be seen at night. The peak of pain due to sickle cell disease is achieved by cold period, that of asthma, in the final phase of nocturnal rest and that of hypertension reaches its maximum at the end of the night resting phase. ${ }^{[45,46]}$

Of the 72 plants identified, 29 must be harvested at a time and/or a specific season in order to have the expected optimal therapeutic activity. Indeed a number of biological activities in the plants including the flowering and the 
synthesis of many secondary metabolites have a moment of peak. In fact, the concentration of active principles in the plant organs evolves following a circannual, circadian even ultradian rhythm. Black et al..$^{[1]}$ found that the phenol content depends on the moment of organ harvest.

The time is important not only in the concentration of active principles in the plant, but also in the susceptibility of the targeted organs during the treatment. ${ }^{[22,47]}$ The 72 plants are used for the preparation of 144 medicinal recipes, 11 of them are active only when administered in the morning, and 19 are active at night. These data are consistent with the requirements of modern drugs, but also with those findings of several authors who highlighted the importance of the timing in the activity of herbal medicines. ${ }^{[22]}$ Thus, for the TH interviewed, the timing is important. Disregard this factor could turn some herbal substances toxic or inactive at best. It should be emphasized that the time of plant collection and administration of drugs can vary from a TH to another. ${ }^{[48]}$

Since the practice of TM is still empirical in Africa, the concept of dose of active ingredients and the mechanism of action are intuitive. This is the main problem with the traditional concoctions in Africa. In fact, the TH only rely on their own experience to prepare their recipes. Raw materials or powders are not weighed nor the volume of water measured, so there is a problem for standardization of the preparations. For the same product, the prescribed dose may vary from one $\mathrm{TH}$ to another or even from one patient to another with the same TH. It is about one to two teaspoons, two to three times daily for the powders and $1 / 2$ to 1 cup, two to three times daily for a decoction or a maceration. A pinch is used for the powder administered by inhalation. Sour products are taken together with food or just meal. The baths are often two times daily, usually morning and evening.

Another problem is the diagnosis of the diseases. In this topic, many $\mathrm{TH}$ also rely on empirical observation to diagnose, thus the risk of misdiagnosis remains in some cases. These are the main goals of CERMETRA, by encouraging $\mathrm{TH}$ for more collaboration with modern medicine. In a recent study, we found that about $75 \%$ of TH confirm their diagnosis with laboratory results but this value is overestimated since these TH are adherents of CERMETRA. ${ }^{[24]}$ This limit could also be due to the patients, since the majority of people treated by TM are poor and thus not able to pay for a laboratory analysis.

\section{CONCLUSION}

This study demonstrated that local specialists in the central region of Togo tend to agree with each other in terms of the plants used to treat diabetes, infertility, and abdominal pains, but cite a much more diverse group of plants to treat problems related to hypertension, sickle cell, and abscess. They also rely on the timing in the plant processing and the administration of herbal remedies. All these findings are based on empirical observation, and laboratory screenings are needed to check the effectiveness of these plants.

\section{ACKNOWLEDGMENTS}

The authors gratefully thank the staff of CERMETRA-RC for their good collaboration.

\section{REFERENCES}

1. Sah AN, Joshi A, Juyal V, Kumar T. Antidiabetic and hypolipidemic activity of Citrus medica Linn. seed extract in streptozotocin induced diabetic rats. Pharmacognosy J 2011;3:80-4.

2. Pandey G, Madhuri S. Some medicinal plants as natural anticancer agents. Pharmacognosy Rev 2009;3:259-63.

3. WHO, "Traditional medicine," 2009. Available from: http:// www.who.int/topics/traditionalmedicine/en. [Last cited on 2011 Aug 20].

4. Karou D, Nadembega WM, Ouattara L, Ilboudo D, Canini A, Nikiéma JB, et al. African ethnopharmacology and new drug discovery. Med Plant Sci Biotechnol 2007;1:61-9.

5. Akomo EF, Zongo C, Karou DS, Obame LC, Atteke C, Savadogo $\mathrm{A}$, et al. In vitro antiplasmodial and antibacterial activities of Canthium multiflorum Schum and Thonn (Rubiacea) extracts. Pak J Biol Sci 2009;12:919-23.

6. Ouattara L, Koudou J, Karou DS, Giacò L, Capelli G, Simpore $\mathrm{J}$, et al. In vitro anti Mycobacterium tuberculosis H37Rv activity of Lannea acida A. Rich. from Burkina Faso. Pak J Biol Sci 2011;14:47-52.

7. Huda AW, Munira MA, Fitrya SD, Salmah M. Antioxidant activity of Aquilaria malaccensis (Thymelaeaceae) leaves. Pharmacognosy Res 2009;1:270-3.

8. Bhujbal SS, Kewatkar SM, More LS, Patil MJ. Antioxidant effects of roots of Clerodendrum serratum Linn. Pharmacognosy Res 2009;1:294-8.

9. Singh M, Khatoon S, Singh S, Kumar V, Rawat AK, Mehrotra S. Antimicrobial screening of ethnobotanically important stem bark of medicinal plants. Pharmacognosy Res 2010;2:254-7.

10. Cock IE, Kukkonen L. An examination of the medicinal potential of Scaevola spinescens: Toxicity, antibacterial, and antiviral activities. Pharmacognosy Res 2011;3:85-94.

11. Mohanty S, Cock IE. Bioactivity of Syzygium jambos methanolic extracts: Antibacterial activity and toxicity. Pharmacognosy Res 2010;2:4-9.

12. De Souza C, Ameyapoh Y, Karou DS, Anani KT, Kpodar ML, Gbeassor M. Assessing market-sold remedies in Lomé (Togo) for hygienic quality. Biotechnol Res Int 2011;2011:572521.

13. Tchacondo T, Karou DS, Batawila K, Agban A, Ouro-Bang'na K, Anani KT, et al. Herbal remedies and their adverse effects in Tem tribe traditional medicine in Togo. Afr J Tradit Complement Altern Med 2011;8:45-60.

14. Moshi MJ, Innocent E, Magadula JJ, Otieno DF, Weisheit A, Mbabazi PK, et al. Brine shrimp toxicity of some plants used as traditional medicines in Kagera Region, north western Tanzania. Tanzan J Health Res 2010;12:63-7. 
15. Black P, Saleem A, Dunford A, Guerrero-Analco J, WalsheRoussel B, Haddad P, et al. Seasonal variation of phenolic constituents and medicinal activities of northern Labrador tea, Rhododendron tomentosum ssp. subarcticum, an Inuit and Cree First Nations traditional medicine. Planta Med 2011;77:1655-62.

16. Vilar J, Ferri P, Chen-Chen L. Genotoxicity investigation of araticum (Annona crassiflora Mart., 1841, Annonaceae) using SOS-Inductest and Ames test. Braz J Biol 2011;71:197-202.

17. Samuels N. Chronotherapy in traditional Chinese medicine. Am J Chin Med 2000;28:419-23.

18. Seki K, Chisaka M, Eriguchi M, Yanagie H, Hisa T, Osada I, et al. An attempt to integrate Western and Chinese medicine: Rationale for applying Chinese medicine as chronotherapy against cancer. Biomed Pharmacother 2005;59 Suppl 1: S132-40.

19. Meng Y, Zhang Z, Liang X, Wu C, Qi G. Effects of combination therapy with amlodipine and fosinopril administered at different times on blood pressure and circadian blood pressure pattern in patients with essential hypertension. Acta Cardiol 2010;65: 309-14.

20. Hermida RC, Ayala DE, Fernández JR, Portaluppi F, Fabbian F, Smolensky MH. Circadian rhythms in blood pressure regulation and optimization of hypertension treatment with ACE inhibitor and ARB medications. Am J Hypertens 2011;24:383-91.

21. Hermida RC, Ayala DE, Mojón A, Fernández JR. Influence of circadian time of hypertension treatment on cardiovascular risk: Results of the MAPEC study. Chronobiol Int 2010;27:1629-51.

22. Reinberg A. Les rythmes biologiques: Mode d'emploie. $2^{\text {nd }} e d$. Paris: Flammarion Médecine Science; 1997. p. 148.

23. Rana S, Mahmood S. Circadian rhythm and its role in malignancy. J Circadian Rhythms 2010;8:3.

24. Karou DS, Tchacondo T, Agassounon Djikpo Tchibozo, AbdoulRahaman S, Anani K, Koudouvo K, et al. Ethnobotanical study of medicinal plants used in the management of diabetes mellitus and hypertension in the Central Region of Togo. Pharm Biol 2011. (corrected proof) DOI: 10.3109/13880209.2011.621959

25. Heinrich M, Ankli A, Frei B, Weimann C, Sticher O. Medicinal plants in Mexico: Healers consensus and cultural importance. Soc Sci Med 1998;47:1859-71.

26. Njoroge NG, Bussmann WR. Ethnotherapeautic management of skin diseases among the Kikuyus of Central Kenya. J Ethnopharmacol 2007;111:303-7.

27. Aburjai T, Hudaib M, Tayyem R, Yousef $M$, Quishawi $M$. Ethnopharmacological survey of medicinal herbs in Jordan, the Ajloun Heights region. J Ethnopharmacol 2006;76:99-103.

28. Hudaib $M$, Mohammad M, Bustanji $Y$, Tayyem R, Yousef $M$, Abuirjeie $M$, et al. Ethnopharmacological survey of medicinal plants in Jordan, Mujib Nature Reserve and surrounding area. J Ethnopharmacol 2008;120:63-71.

29. Koudouvo K, Karou DS, Kokou K, Essien K, Aklikokou K, Glitho IA, et al. An ethnobotanical study of antimalarial plants in Togo Maritime Region. J Ethnopharmacol 2011;134:183-90.

30. Nadembega P, Boussim JI, Nikiema JB, Poli F, Antognoni F. Medicinal plants in Baskoure, Kourittenga Province, Burkina Faso: An ethnobotanical study. J Ethnopharmacol 2011;133: 378-95.

31. Maroyi A. An ethnobotanical survey of medicinal plants used by the people in Nhema communal area, Zimbabwe. J Ethnopharmacol 2011;136:347-54.

32. Telefo PB, Lienou LL, Yemele MD, Lemfack MC, Mouokeu C, Goka CS, et al. Ethnopharmacological survey of plants used for the treatment of female infertility in Baham, Cameroon. $J$ Ethnopharmacol 2011;136:178-87.

33. Nacoulma-Ouédraogo OG. Medicinal plants and traditional medical practices in Burkina Faso. Case of the Mossi central plateau State Thesis, University of Ouagadougou; 1996.

34. Koné WM, Kamanzi Atindehou K, Terreaux C, Hostettmann K, Traoré D, Dosso M. Traditional medicine in North Côte-d'Ivoire: Screening of 50 medicinal plants for antibacterial activity. J Ethnopharmacol 2004;93:43-9.

35. Inngjerdingen K, Nergård CS, Diallo D, Mounkoro PP, Paulsen BS. An ethnopharmacological survey of plants used for wound healing in Dogonland, Mali, West Africa. J Ethnopharmacol 2004;92:233-44.

36. Magassouba FB, Diallo A, Kouyaté M, Mara F, Mara O, Bangoura $\mathrm{O}$, et al. Ethnobotanical survey and antibacterial activity of some plants used in Guinean traditional medicine. J Ethnopharmacol 2007; 114:44-53.

37. Karou D, Dicko MH, Sanon S, Simpore J, Traore SA. Antimalarial activity of Sida acuta BURMF L. (Malvaceae) and Pterocarpus erinaceus POIR (Fabaceae). J Ethnopharmacol 2003;89:291-4.

38. Karou D, Dicko MH, Simpore J, Traore AS. Antioxidant and antibacterial activities of polyphenols from ethnomedicinal plants of Burkina Faso. Afr J Biotechnol 2005;4:823-8.

39. Abdelgaleil SA, Iwagawa T, Doe M, Nakatani M. Antifungal limonoids from the fruits of Khaya senegalensis. Fitoterapia 2004;75:566-72.

40. Gbolade AA. Inventory of antidiabetic plants in selected districts of Lagos State, Nigeria. J Ethnopharmacol 2009;121:135-9.

41. Okoli AS, Iroegbu CU. Evaluation of extracts of Anthocleista djalonensis, Nauclea latifolia and Uvaria afzalii for activity against bacterial isolates from cases of non-gonococcal urethritis. J Ethnopharmacol 2004;92:135-44.

42. Chah KF, Eze CA, Emuelosi CE, Esimone CO. Antibacterial and wound healing properties of methanolic extracts of some Nigerian medicinal plants. J Ethnopharmacol 2006;104:164-7.

43. Maiga A, Diallo D, Fane S, Sanogo R, Paulsen BS, Cisse B. A survey of toxic plants on the market in the district of Bamako, Mali: Traditional knowledge compared with a literature search of modern pharmacology and toxicology. $J$ Ethnopharmacol 2005;96:183-93.

44. Karou DS, Tchacondo T, Ilboudo D, Simpore J. Sub-Saharan Rubiaceae: A review of their traditional usages, phytochemistry and biological activities. Pak J Biol Sci 2011;14:149-69.

45. Munger MA, Kenney JK. A chronobiologic approach to the pharmacotherapy of hypertension and angina. Ann Pharmacother 2000;34:1313-9.

46. Richardson V. Rabbits: Health, husbandry and disease. Blackwell Science Inc.; 2000. p. 178.

47. Zaslavskaia RM, Buniatian ND, Sergeev SV, Lukashev AM, Lednev OA. Efficacy of traditional therapy and chronotherapy using prestarium in elderly patients with polymorbid syndrome. Klin Med (Mosk) 2010;88:71-2.

48. Agassounon Dikpo Tchibozo M, Toukourou F, de Souza C, Gbeassor M. Cytotoxic, antiviral, antibacterial and antifungal six plants used in traditional medicine in Benin. Rev Méd Pharm Afr 2007;20:115-24.

Cite this article as: Tchacondo T, Karou SD, Agban A, Bako M, Batawila K, Bawa ML, Gbeassor M, de Souza C. Medicinal plants use in central Togo (Africa) with an emphasis on the timing. Phcog Res 2012;4:92-103.

Source of Support: Nil, Conflict of Interest: None declared. 